\title{
Specification and Complexity of Strategic-Based Reasoning Using Argumentation
}

\author{
Mohamed Mbarki ${ }^{1}$, Jamal Bentahar ${ }^{2}$, and Bernard Moulin ${ }^{1}$ \\ ${ }^{1}$ Laval University, Department of Computer Science and Software Engineering, \\ Canada \\ \{mohamed.mbarki, bernard.moulin\}@ift.ulaval.ca \\ ${ }^{2}$ Concordia University, Concordia Institute for Information Systems Engineering \\ (CIISE), Canada \\ bentahar@ciise.concordia.ca
}

\begin{abstract}
In this paper, we propose a new strategic and tactic reasoning for agent communication. This reasoning framework is specified using argumentation theory combined to a relevance theory. Strategic reasoning enables agents to decide about the global communication plan in terms of the macro-actions to perform in order to achieve the main conversational goal. Tactic reasoning, on the other hand, allows agents to locally select, at each moment, the most appropriate argument according to the adopted strategy. Previous efforts at defining and formalizing strategies for argumentative agents have often neglected the tactic level and the relation between strategic and tactic levels. In this paper, we propose a formal framework for strategic and tactic reasoning for rational communicating agents and the relation between these two kinds of reasoning. Furthermore, we address the computational complexity of this framework and we argue that this complexity is in the same level of the polynomial hierarchy than the complexity of the strategic-free argumentation reasoning.
\end{abstract}

\section{Introduction}

Recent years have seen an increasing interest in agent communication. Using argumentation theories in this domain seems a promising way to develop more flexible and efficient agent communication mechanisms [1/3/4/14/16/28]. The idea is to provide agents with reasoning capabilities allowing them to decide about the appropriate communicative acts to perform in order to achieve some conversational goals in different dialogue types [18/19|22|23|26].

In order to improve the agent communication efficiency, we propose in this paper a formal framework addressing strategic and tactic issues. A strategy is defined as a global cognitive representation of the means of reaching some goals 33. Tactic is basically the mean to reach the aims fixed at the strategic level [20. For example, according to Moore [20, maintaining focus of the dispute in a persuasive dialogue, and building a point of view or destroying the opponent's one refer to strategy, whereas selecting methods to fulfill these two objectives 
refers to tactic. In our framework, the agents' strategic and tactic reasoning is based upon their argumentative capabilities. Agents use this reasoning in order to achieve their conversational goals. Strategic reasoning allows agents to plan the global line of communication in terms of the sub-goals to achieve, whereas tactic reasoning allows them to locally select, at each moment, the most appropriate argument according to the adopted strategy. In other words, strategy is considered at the global level (in which direction the communication can advance) and the tactics are considered at the local level (which move to be selected next).

In recent years, some significant proposals have explored the strategic reasoning of argumentative agents 2[15/27/29. However, the tactical reasoning has often been neglected or simplified to a private preference policy like in [15. In addition, as outlined in 10, the problem of coming up with an optimal communication strategy that ensures beneficial interaction outcomes for the participating agents is still an open problem. We think that an efficient agent communication requires to address both the strategic and tactic levels and the relation between these two levels. The objective of this paper is to investigate this issue for argumentative-based agent communication. Our contribution starts by formalizing strategic and tactic reasoning and the relation between them using a management theory. At the tactical level, we develop a theory allowing agents to select the most relevant argument at each moment according to the adopted strategy. In addition, our approach enables agents to take into account the conversation context and to be able to backtrack if some choices are not appropriate.

Paper overview. In Section 2, we introduce the fundamental ideas of our agent communication approach based on social commitments and arguments. In Section 3, we present the strategic level of our framework and its relation with the tactic level. In Section 4, we present the tactic reasoning. In Section 5, we illustrates our ideas by an example. In Section 6, we discuss the computational complexity of our framework. In Section 7, we compare our framework to related work and conclude the paper.

\section{Agent Communication Approach}

Our agent communication approach is based on the philosophical notion of social commitments (SCs) 32. A SC is an engagement made by an agent (called the debtor), that some fact is true or that some action will be performed. This commitment is directed to a set of agents (called creditors). A SC is an obligation in the sense that the debtor must respect and behave in accordance with this commitment. Commitments are social in the sense that they are expressed publicly and governed by some rules. This means that they are observable by all the participants. The main idea is that a speaker is committed to a statement when he made this statement or when he agreed upon this statement made by another participant and acts accordingly. For simplification reasons, we suppose that we have only one creditor. Thus, we denote a SC as follows: 
$S C\left(A g_{1}, A g_{2}, t, \varphi\right)$ where $A g_{1}$ is the debtor, $A g_{2}$ is the creditor, $t$ is the time associated with the commitment, and $\varphi$ its content. Logically speaking, a SC is a public propositional attitude. The content of a $\mathrm{SC}$ can be a proposition or an action. A detailed taxonomy of the SCs is presented in [5] and their logical semantics is developed in [6].

In order to model the dynamics of conversations in our framework, we interpret a speech act as an action performed on a SC or on a SC content. A speech act is an abstract act that an agent, the speaker, performs when producing an utterance $U$ and addressing it to another agent, the addressee [31. According to speech act theory 31, the primary units of meaning in the use of language are not isolated propositions but rather speech acts of the type called illocutionary acts. Assertions, questions, orders and declarations are examples of these illocutionary acts. In our framework, a speech act can be defined using BNF notation as follows.

Definition 1 (Speech Acts). $S A\left(i_{k}, A g_{1}, A g_{2}, t_{u}, U\right)=_{\text {def }}$

$$
\begin{aligned}
& A c t\left(A g_{1}, t_{u}, S C\left(A g_{1}, A g_{2}, t, \varphi\right)\right) \\
& \mid A c t-\operatorname{cont}\left(A g_{1}, t_{u}, S C\left(A g_{i}, A g_{j}, t, \varphi\right)\right) \\
& \mid A c t\left(A g_{1}, t_{u}, S C\left(A g_{1}, A g_{2}, t, \varphi\right)\right) \& \\
& A c t-\operatorname{cont}\left(A g_{1}, t_{u}, S C\left(A g_{i}, A g_{j}, t, \varphi\right)\right)
\end{aligned}
$$

where $S A$ is the abbreviation of "Speech Act", $i_{k}$ is the identifier of the speech act, $A g_{1}$ is the speaker, $A g_{2}$ is the addressee, $t_{u}$ is the utterance time, $U$ is the utterance, Act indicates the action performed by the speaker on the commitment: Act $\in\{$ Create, Withdraw, Violate, Satisfy\}, Act-cont indicates the action performed by the speaker on the commitment content: Act-cont $\in\{$ Acceptcont, Refuse-cont, Challenge-cont, Justify-cont, Defend-cont, Attack-cont $\}$, $i, j \in\{1,2\}, i \neq j$, the meta-symbol "\&" indicates the logical conjunction between actions performed on social commitments and social commitment contents.

The definiendum $S A\left(i_{k}, A g_{1}, A g_{2}, t_{u}, U\right)$ is defined by the definiens $A c t\left(A g_{1}, t_{u}, S C\left(A g_{1}, A g_{2}, t, \varphi\right)\right)$ as an action performed by the speaker on its SC. The definiendum is defined by the definiens $\operatorname{Act} \operatorname{cont}\left(A g_{1}, t_{u}, S C\left(A g_{i}, A g_{j}, t, \varphi\right)\right)$ as an action performed by the speaker on the content of its $\mathrm{SC}(i=1, j=2)$ or on the content of the addressee's SC $(i=2, j=1)$. Finally, the definiendum is defined as an action performed by the speaker on its SC and as an action performed by the speaker on the content of its $\mathrm{SC}$ or on the content of the addressee's SC. These actions are similar to the moves proposed in [30.

We notice here that using a social (public) approach as a theoretical foundation does not mean that agents do not reason on their private mental states or on the addressees' mental states (beliefs, intention, etc.). According to Definition 1 , this public approach is used at the semantical level in order to interpret communicative acts as social commitments and not as mental states (see [6]7] for more details about the public semantics). Public and mental (private) approaches are not contradictory, but rather, they are complementary. In our framework, agents reason on SCs and on their beliefs about the addressees' beliefs and preferences 
(see Section 4.2). These beliefs are not public, but they can, for example, be inferred from past interactions.

Our approach is also based on argumentation. Several argumentation theories and frameworks have been proposed in the literature (see for example [9[17/25]). An argumentation system essentially includes a logical language $£$, a definition of the argument concept, a definition of the attack relation between arguments, and finally a definition of acceptability. We use the following definitions from [1]. Here $\Gamma$ indicates a possibly inconsistent knowledge base with no deductive closure, and $\vdash$ stands for classical inference.

Definition 2 (Argument). An argument is a pair $(H, h)$ where $h$ is a formula of $£$ and $H$ a subset of $\Gamma$ such that: $i) H$ is consistent, ii) $H \vdash h$ and iii) $H$ is minimal, so that no subset of $H$ satisfying both $i$ and ii exists. $H$ is called the support of the argument and $h$ its conclusion.

Definition 3 (Attack). Let $(H, h),\left(H^{\prime}, h^{\prime}\right)$ be two arguments. $\left(H^{\prime}, h^{\prime}\right)$ attacks $(H, h)$ iff $H^{\prime} \vdash \neg h$. In other words, an argument is attacked if and only if there exists an argument for the negation of its conclusion.

The link between commitments and arguments enables us to capture both the public and reasoning aspects of agent communication. This link is explained as follows. Before committing to some fact $h$ being true (i.e. before creating a commitment whose content is $h$ ), the speaker agent must use its argumentation system to build an argument $(H, h)$. On the other side, the addressee agent must use its own argumentation system to select the answer it will give (i.e. to decide about the appropriate manipulation of the content of an existing commitment). For example, an agent $A g_{1}$ accepts the commitment content $h$ proposed by another agent $A g_{2}$ if it is able to build an argument supporting this content from its knowledge base. If $A g_{1}$ has an argument $\left(H^{\prime}, \neg h\right)$, then it refuses the commitment content proposed by $\mathrm{Ag}_{2}$. However, how agents can select the most appropriate argument at a given moment depends on its tactic. This aspect is detailed in Section 4. The social relationship that exists between agents, their reputations and trusts also influence the acceptance of the arguments by agents. However, this aspect will not be dealt with in this paper. The argumentation relations that we use in our model are thought of as actions applied to commitment contents. The set of these relations is: $\{$ Justify,Defend, Attack\}.

In order to implement this communication model, we use an agent architecture composed of three layers: the mental layer, the social layer, and the reasoning layer. The mental layer includes beliefs, desires, goals, etc. The social layer captures social concepts such as SCs, conventions, roles, etc. Agents must use their reasoning capabilities to reason about their mental states before acting on SCs. The agent's reasoning capabilities are represented by the reasoning layer using an argumentation system. Our conversational agent architecture also involves general knowledge, such as knowledge about the conversation subject. Agents can also reason about their preferences in relation to beliefs. The idea is to capture the fact that some facts are more strongly believed. For this reason, we assume, like in [1, that any set of facts has a preference order over it. We suppose that this ordering derives from the fact that the agent's knowledge base 
denoted by $\Gamma$ is stratified into non-overlapping sets $\Gamma_{1}, \ldots, \Gamma_{n}$ such that facts in $\Gamma_{i}$ are all equally preferred and are more preferred than those in $\Gamma_{j}$ where $i<j$. We can also define the preference level of a subset of $\Gamma$ whose elements belong to different non-overlapping sets as follows.

Definition 4 (Preference Level). The preference level of a nonempty subset $\gamma$ of $\Gamma$ denoted by level $(\gamma)$ is the number of the highest numbered layer which has a member in $\gamma$.

Example 1. Let $\Gamma=\Gamma_{1} \cup \Gamma_{2}$ with $\Gamma_{1}=\{a, b\}$ and $\Gamma_{2}=\{c, d\}$ and $\gamma=\{a\}$ and $\gamma^{\prime}=\{a, d\}$. We have: level $(\gamma)=1$ and $\operatorname{level}\left(\gamma^{\prime}\right)=2$.

\section{$3 \quad$ Strategic Reasoning}

According to the theory of constraints proposed by Goldratt [13, the common view about strategy is that of setting the high objectives of an initiative. The strategy dictates the direction of all activities. Tactics, on the other hand, are the chosen types of activities needed to achieve the objectives. Indeed, tactics allow us to implement and accomplish the strategy. In management, a strategic plan defines the mission, vision and value statements of an enterprize. Once objectives are defined, alternative strategies can be evaluated. While a goal or an objective indicates "what" is to be achieved, a strategy indicates "how" that achievement will be realized. Strategies, therefore, depend on goals and objectives. Tactics are the steps involved in the execution of the strategy.

Our strategic and tactic framework for agent communication is based on this vision. In this framework, the dialogue strategy is defined in terms of the subgoals to be achieved in order to achieve the final conversational goal. The subgoals represents the macro-actions to be performed. This reflects the global vision and the direction of the dialogue. The strategy has a dynamic nature in the sense that the sub-goals can be elaborated while the dialogue advance. The strategy can also be adjusted when more information becomes available. The tactics represent the micro-actions to be performed in order to achieve each elaborate (elementary) sub-goal. This reflects the local vision of the dialogue. A tactic is succeeded when the sub-goal is achieved, and the strategy is succeeded when all the involved tactics are succeeded, which means that the final goal is achieved. Fig. 11illustrates the strategic and tactic levels in our framework.

Indeed, in multi-agent systems, agents are designed to accomplish particular tasks. Each agent has its own domain and a certain goals to achieve. We call this kind of goals: operational goals. These agents often have to interact with each other in order to achieve some sub-goals of the operational goals. These sub-goals generate what we call conversational goals. In our framework, we distinguish between these two types of goals. In the same way, we distinguish between domain constraints, called operational constraints, and conversational constraints called criterions. Time and budget constraints are examples of operational constraints, and respecting the religious and ideological believes of the addressee is an example of criterions. In our framework, a dialogue strategy depends on the conversational goal, operational constraints and criterions. Operational constraints and 


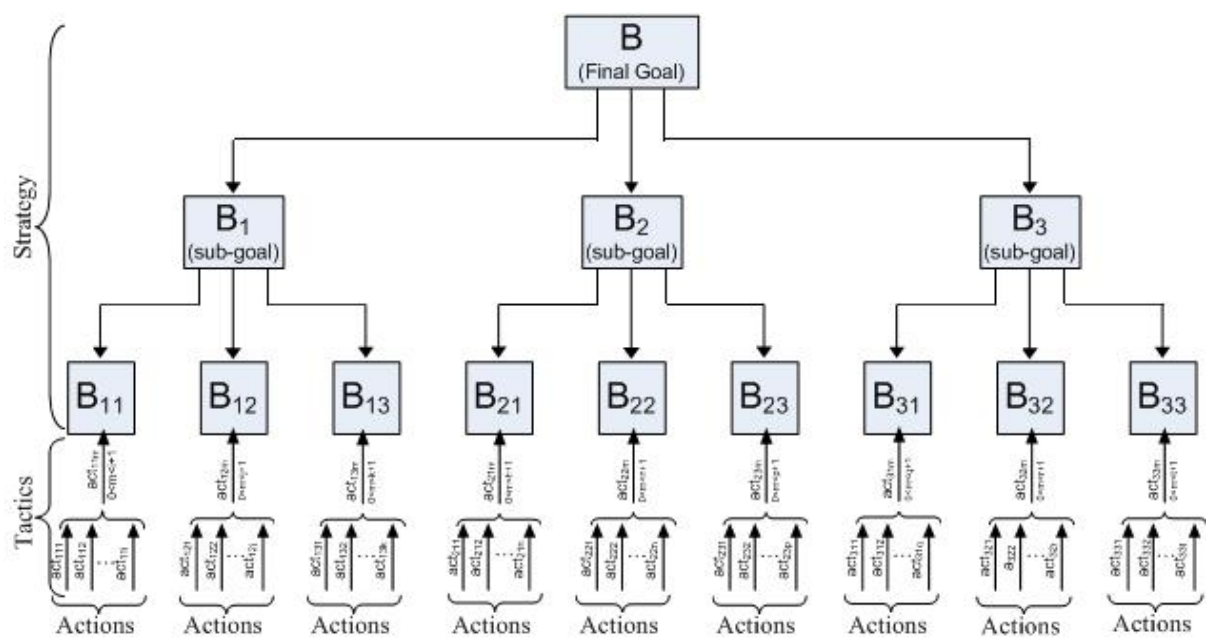

Fig. 1. Strategy and tactics in our framework

criterions also reflect the factors that may influence the strategy design: goals, domain, agents' capabilities, agents' values, protocol, counterparts, agents' resources, and alternatives [27]. Domain, agents' capabilities, and agents' values are operational constraints. Protocol, counterparts, agents' resources, and alternatives are criterions.

The initiative agent must build a global and initial strategy before starting the conversation. A strategy allows an agent to decide about the main sub-goals to be fixed in order to achieve the conversational goal according to a set of operational constraints and conversational criterions. To achieve the same conversational goal, an agent can have several alternative strategies depending on the sub-set of operational constraints and the sub-set of criterions the agent decide to satisfy. The conversational goal, sub-goals, operational constraints and criterions can be expressed in a logical language. The set of operational constraints and the set of criterions can be inconsistent. However, the sub-set of operational constraints and the sub-set of criterions the agent decide to satisfy should be consistent. We define a strategy as a function that associates to a goal and a sub-set of operational constraints and a sub-set of criterions a set of goals (sub-goals).

Definition 5 (Strategy). Let $\mathcal{B}$ be a set of goals, Ctr be a set of operational constraints, and $\mathrm{Cr}$ be a set of conversational criterions. A strategy is a function: $S t r: \mathcal{B} \times 2^{C t r} \times 2^{C r} \rightarrow 2^{\mathcal{B}}$

Strategies are dynamic in nature. Agents should adjust the adopted dialogue strategy while the conversation progresses. This can be achieved by taking into account the new constraints and criterions that can appear during the conversation. In this case, the new constraints and criterions to be satisfied should be consistent with the initial sub-set of constraints and criterions selected to 
be satisfied. Thus, agents can apply the strategy function (Str) each time new constraints and criterions are added. This enables agents to decide about the subgoals to be achieved of each already fixed sub-goal. In Fig. 1 this is illustrated by the different levels: from a level $i$ to a level $i+1$ (we suppose that the level in which we have the main or final goal is the lower one). We notice here that the set of criterions can progress with the dialogue, whereas the set of operational constraints is generally more stable.

Example 2. Let us suppose that: $C t r=\left\{x_{0}, x_{1}, x_{2}\right\}$ and $C r=\left\{y_{0}, y_{1}\right\}$. Let $B \in \mathcal{B}$ be the conversational goal, and $S C t r$ and $S C r$ be two sub-sets of $C t r$ and $\mathrm{Cr}$ representing the constraints and criterions selected to be satisfied. We suppose that: $S C t r=\left\{x_{0}, x_{1}\right\}$ and $S C r=\left\{y_{1}\right\}$. We can have at a first time (level 0): $\operatorname{Str}(B, S C t r, S C r)=\left\{B_{1}, B_{2}, B_{3}\right\}$. At a second time (level 1), we suppose that: $S C r=S C r \cup\left\{y_{2}\right\}$. Thus, by applying the $S t r$ function on $B_{1}$, we can obtain: $\operatorname{Str}\left(B_{1}, S C t r, S C r\right)=\left\{B_{11}, B_{12}, B_{13}\right\}$.

This example illustrates how the strategy can influence the dialogue by deciding about the sub-goals to achieve in order to achieve the main conversational goal. The dialogue advance, on the other hand, influences the strategy by taking into account the new operational constraints and criterions. In the case where the new constraints and criterions are inconsistent with the initial selected ones, the adopted strategy should be completely or partially changed. The strategy should be completely changed if the main goal is changed. However, if only one of the sub-goals is changed, the strategy should be partially changed.

In our framework, agents start by using the strategic reasoning to build the general line of communication. This is reflected by applying the function Str on the main conversational goal. Thereafter, strategic reasoning and tactic reasoning are used in parallel. The link between strategy and tactics is that each tactic is related to a sub-goal fixed by the strategy. The execution of a tactic allows the execution, the evolution, and the adaptation of the strategy. For example, if the tactic does not allow the achievement of a sub-goal, the strategy should be adapted to fix another sub-goal.

\section{Tactic Reasoning}

In this section, we present our theory of the tactical reasoning for argumentationbased communicative agents. As illustrated in Fig. 1, tactics allow agents to select from a set of actions, one action in order to achieve a sub-goal fixed by the adopted strategy. The purpose of our theory is to guarantee that the selected action is the most appropriate one according to the current context. In the rest of this paper, the actions we consider are arguments that agents use to support their points of view or attack the opponent's point of view. The most appropriate action is then the most relevant argument. This enables agents to be more efficient in their argumentation. Our theory is based on the relevance of arguments. 


\subsection{Relevance of Arguments}

The most significant attempts to formalize relevance have been done by van Rooy [34] and Fleger [12]. van Rooy supposes that the relevance of a communication act in purely competitive dialogues depends on its argumentative force in a given context. The argumentative force of a proposition with respect to a hypothesis is defined by a probability function, which assigns a value to a proposition. This value represents the probability that this proposition is true. However, van Rooy does not specify how we can assign probabilities to different propositions. Fleger's proposal is based on the proof theory of minimality. It considers that an argument is irrelevant if it is not in relation to the conversation subject (or problem to be solved) or if it contains useless premises. This notion of relevance takes into account only the agent's knowledge base without considering the conversation context. In addition, the minimality concept is not related to the notion of relevance, but it is a part of arguments definition.

In our framework, we define the relevance of an argument according to the conversation context. Our objective is to allow agents to select the most relevant argument at a given moment by taking into account not only the last communicative act, but also the previous acts. The idea is to provide a solution allowing backtracking. This means that, an agent selects one among a set of possible arguments represented as a tree. If the choice proves to be incorrect because the selected argument is not accepted by the addressee agent and cannot be defended, the agent can backtrack or restart at the last point of choice and can try another argument, which is represented by trying another path in the tree. The arguments are ordered according to their relevance. We call this process arguments selection mechanism.

\subsection{Arguments Selection Mechanism}

Let $L$ be a logical language. The conversation context for an agent $A g_{1}$ committed in a conversation with another agent $A g_{2}$ is defined as follows.

Definition 6 (Context). The conversation context for an agent $A g_{1}$ (the speaker) committed in a conversation with an agent $A_{2}$ (the addressee) is a 5-tuple $C_{A g_{1}, A g_{2}}=\left\langle S, s, \mathcal{P}_{A g_{1}, A g_{2}}, K D\right\rangle$ where:

- $S$ is a formula of $L$ representing the conversation subject that corresponds to the conversational goal,

- $s$ is a formula of $L$ representing the argument on which the speaker should act,

- $\mathcal{P}_{A g_{1}, A g_{2}}$ is the set of $A g_{1}$ 's beliefs about $A g_{2}$ 's beliefs $\mathcal{P}_{A g_{1}, A g_{2}}^{b e l}$ and about $A g_{2}$ 's preferences $\mathcal{P}_{A g_{1}, A g_{2}}^{\text {pref }}$. Thus $\mathcal{P}_{A g_{1}, A g_{2}}=\mathcal{P}_{A g_{1}, A g_{2}}^{\text {bel }} \cup \mathcal{P}_{A g_{1}, A g_{2}}^{\text {pref }}$,

- $K D$ is the knowledge that the two agents share about the conversation.

$K D$ can contain results or laws related to the domain that are already proved. In addition, all information on which the two agents agree during the current 
conversation is added to $K D$. For example, the accepted arguments are added to $K D$. We also assume that $K D \cap \mathcal{P}_{A g_{1}, A g_{2}}=\varnothing$.

In the context $C_{A g_{1}, A g_{2}}$, formula $s$ should be relevant for subject $S$ in the sense that there is a logical relation between the two formulas. This relation represents the link between tactic and strategy. The idea is that the current action (at the tactic level) is related to a sub-goal, which is fixed by the strategy. The current argument can attack or support the formula representing the subgoal. In order to define this logical relation between $S$ and $s$, we introduce the notion of argumentation tree and the notion of path that we define as follows.

Definition 7 (Argumentation Tree). Let $A$ be the set of participating agents and $A R$ be the set of arguments used by the agents in the dialogue. An argumentation tree $T$ is a 2-tuple $T=\langle N, \rightarrow\rangle$ where:

- $N=\left\{\left(A g_{i},(H, h)\right) \mid A g_{i} \in A,(H, h) \in A R\right\}$ is the set of nodes. Each node is described as a pair $\left(A g_{i},(H, h)\right)$, which indicates that the argument $(H, h)$ is used by the agent $A g_{i}$,

- $\rightarrow \subseteq N \times N$ is a relation between nodes. We write $n_{0} \rightarrow n_{1}$ instead of $\left(n_{0}, n_{1}\right) \in \rightarrow$ where $\left\{n_{0}, n_{1}\right\} \subseteq N$. The relation $\rightarrow$ is defined as follows: $\left(A g_{1},(H, h)\right) \rightarrow\left(A g_{2},\left(H^{\prime}, h^{\prime}\right)\right)$ iff $A g_{1} \neq A g_{2}$ and $\left(H^{\prime}, h^{\prime}\right)$ attacks $(H, h)$ (see definition 3 ).

This notion of argumentation tree is close to the notion of argument tree introduced in [8] and to the notion of abstract dispute tree used in [11. The main difference between our argumentation tree notion and these two notions is that the first one is used to formalize the logical relation between the conversation subject $S$ and the current argument $s$ and not to illustrate the dialectical proof and the acceptance of arguments. In addition, our argumentation tree is used to illustrate the backtracking process which is not dealt with in [8] and in [11.

We associate each (argumentative) conversation to an argumentation tree. The root of such an argumentation tree is the initial node $n_{0}=\left(A g_{i},(H, S)\right)$ where $A g_{i}$ is the initiating agent $\left(A g_{i} \in A\right)$ and $(H, S)$ is the argument supporting the conversation subject (or the conversation goal).

Definition 8 (Path). Let $T=\langle N, \rightarrow\rangle$ be an argumentation tree. A path in $T$ is a finite sequence of nodes $n_{0}, n_{1}, \ldots, n_{m}$ such that $\forall i \quad 0 \leq i<m: n_{i} \rightarrow n_{i+1}$.

Proposition 1. Let $C_{A g_{1}, A g_{2}}=\left\langle S, s, \mathcal{P}_{A g_{1}, A g_{2}}, K D\right\rangle$ be a conversation context and $A=\left\{A g_{1}, A g_{2}\right\}$ be the set of participating agents. There is a logical relation between $S$ and $s$ in the context $C_{A g_{1}, A g_{2}}$ iff there is a path in the argumentation tree associated with the conversation between the root and the current node $n_{m}=$ $\left(A g_{i},\left(H^{\prime}, s\right)\right)$ where $i \in\{1,2\}$ and $\left(H^{\prime}, s\right)$ is the argument supporting $s$.

The existence of a path in the tree between the root and the current argument means that this argument defends or attacks directly or indirectly the conversation subject. Thus, independently on the path, there is a logical relation between $S$ and $s$. 
In our approach, we first distinguish between relevant and irrelevant arguments in a given context. This distinction allows agents to eliminate at each argumentation step irrelevant arguments before ordering the relevant arguments in order to select the most relevant one.

Definition 9 (Irrelevant Argument). Let $C_{A g_{1}, A g_{2}}=\left\langle S, s, \mathcal{P}_{A g_{1}, A g_{2}}, K D\right\rangle$ be a conversation context, $A$ be the set of participating agents, $T=\langle N, \rightarrow\rangle$ be the argumentation tree associated to the conversation, and $\left(A g_{i},(H, h)\right)$ be a node in $T$ where $i \in\{1,2\} .(H, h)$ is irrelevant in the context $C_{A g_{1}, A g_{2}}$ iff:

1. There is no path between the node $\left(A g_{i},(H, h)\right)$ and the root of $T$ or;

2. $\exists x \in K D: H \vdash \neg x$.

The first clause states that the argument does not address the conversation subject. The second clause states that the argument contradicts the shared knowledge. We notice here that $K D$ is a knowledge base that changes during the conversation. Thus, an argument built at a step $t_{i}$ can become irrelevant at a later step $t_{j}$ if it contradicts the new information accepted by the agent. In these two cases, the argument is irrelevant and the agent can not use it. Irrelevant arguments must be removed from the set of arguments that the agent can use at a given step of the conversation. This set, called the set of potential arguments, is denoted by $P A$.

In Section 2, we emphasized the fact that agents can have private preferences about different knowledge (see definition 4). Therefore, they can have private preferences about arguments. This preference relation denoted by $(H, h) \ll_{\text {pref }}^{A g_{i}}$ $\left(H^{\prime}, h^{\prime}\right)$ means that agent $A g_{i}$ prefers the argument $\left(H^{\prime}, h^{\prime}\right)$ to the argument $(H, h)$. We define this relation as follows.

Definition 10 (Preference). Let $(H, h)$ and $\left(H^{\prime}, h^{\prime}\right)$ be two arguments. $(H, h) \ll_{\text {pref }}^{A g_{i}}\left(H^{\prime}, h^{\prime}\right)$ iff level $\left(H^{\prime}\right) \leq \operatorname{level}(H)$.

Because $\leq$ is an ordering relation, the preference relation $\ll_{p r e f}^{A g_{i}}$ is reflexive, antisymmetric, and transitive. Agents may also have favorites among their arguments. How an agent favors an argument over others depends on the dialogue type. For example, in a persuasive dialogue, an agent can favor arguments having more chances to be accepted by the addressee. In order to characterize this notion, we introduce the notion of weight of an argument. The weight of an argument $(H, h)$ compared to another argument $\left(H^{\prime}, h^{\prime}\right)$ in the context $C_{A g_{1}, A g_{2}}=\left\langle S, s, \mathcal{P}_{A g_{1}, A g_{2}}, K D\right\rangle$ is denoted by $W_{(H, h) /\left(H^{\prime}, h^{\prime}\right)}^{\mathcal{P}_{A g_{1}, A g_{2}}}$ and is evaluated according to the following algorithm:

According to this algorithm, the weight of an argument $(H, h)$ compared to another argument $\left(H^{\prime}, h^{\prime}\right)$ is incremented by 1 each time $A g_{1}$ believes that $A g_{2}$ prefers a knowledge in $H$ to a knowledge in $H^{\prime}$. Indeed, each element of $H$ is compered once to each element of $H^{\prime}$ according to the preference relation. Consequently, the weight of an argument is finite because $H$ and $H^{\prime}$ are finite sets. 


\section{Algorithm 1 (Evaluation of an Argument compared to Another One)}

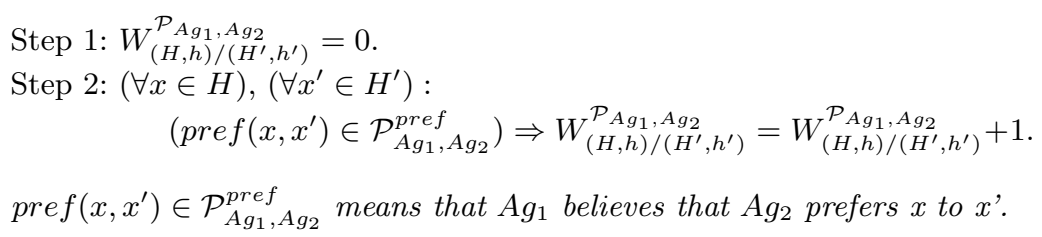

The favorite relation is denoted by $\preceq_{\text {fav }}^{\mathcal{P}_{A g_{1}, A g_{2}}}$ and the strict favorite relation is denoted by $\prec_{f a v}^{\mathcal{P}_{A g_{1}, A g_{2}}} .(H, h) \preceq_{f a v}^{\mathcal{P}_{A g_{1}, A g_{2}}}\left(H^{\prime}, h^{\prime}\right)$ means that agent $A g_{1}$ favors the argument $\left(H^{\prime}, h^{\prime}\right)$ over the argument $(H, h)$ according to $\mathcal{P}_{A g_{1}, A g_{2}}$. This relation is defined as follows.

Definition 11 (Favorite Argument). Let $C_{A g_{1}, A g_{2}}=\left\langle S, s, \mathcal{P}_{A g_{1}, A g_{2}}, K D\right\rangle$ be a conversation context and $(H, h)$ and $\left(H^{\prime}, h^{\prime}\right)$ be two arguments in the context $C_{A g_{1}, A g_{2}}$. We have :

$$
\begin{aligned}
& (H, h) \preceq_{f a v}^{\mathcal{P}_{A g_{1}, A g_{2}}} \quad\left(H^{\prime}, h^{\prime}\right) \quad \text { iff } \quad W_{(H, h) /\left(H^{\prime}, h^{\prime}\right)}^{\mathcal{P}_{A g_{1}, A g_{2}}} \leq W_{\left(H^{\prime}, h^{\prime}\right) /(H, h)}^{\mathcal{P}_{A g_{1}, A g_{2}}}, \\
& (H, h) \underset{\text { fav }}{\mathcal{P}_{A g_{1}, A g_{2}}}\left(H^{\prime}, h^{\prime}\right) \quad \text { iff } \quad W_{(H, h) /\left(H^{\prime}, h^{\prime}\right)}^{\mathcal{P}_{A g_{1}, A g_{2}}}<W_{\left(H^{\prime}, h^{\prime}\right) /(H, h)}^{\mathcal{P}_{A g_{1}}, A g_{2}} .
\end{aligned}
$$

In order to allow agents to select the most relevant argument in a conversation context, we introduce an ordering relation between relevant arguments. This ordering relation depends on the adopted strategy and is based on the notion of the risk of failure of an argument. This notion of risk is subjective and there are several heuristics to evaluate the risk of an argument. In this paper we use a heuristic based on the fact that $K D$ contains certain knowledge and $\mathcal{P}_{A g_{1}, A g_{2}}$ contains uncertain beliefs. We formally define this notion as follows.

Definition 12 (Risk of Failure of an Argument). Let $C_{A g_{1}, A g_{2}}=$ $\left\langle S, s, \mathcal{P}_{A g_{1}, A g_{2}}, K D\right\rangle$ be a conversation context and $(H, h)$ be a relevant argument in the context $C_{A g_{1}, A g_{2}}$. The risk of failure of $(H, h)$ denoted by risk $((H, h))$ is the sum of the risks of failure of all the formulas included in $H$. The risk of failure of a formula $q$ denoted by risk $(q)$ is defined as follows:

- if $q \in K D$ then $\operatorname{risk}(q)=v_{1}$.

- if $q \in \mathcal{P}_{A g_{1}, A g_{2}}$ then $\operatorname{risk}(q)=v_{2}$.

- otherwise $\operatorname{risk}(q)=v_{3}$.

Where $v_{1}<v_{2}<v_{3}$ and $v_{1}, v_{2}, v_{3} \in \mathbb{R}$.

Values $v_{1}, v_{2}$ and $v_{3}$ should be instantiated according to the dialogue type and the confidence level of the beliefs included in $\mathcal{P}_{A g_{1}, A g_{2}}$. For example, in a persuasive dialogue and if we consider that $K D$ contains certain knowledge, we may have $v_{1}=0, v_{2}=0.25, v_{3}=0.5$. If the confidence level of $\mathcal{P}_{A g_{1}, A g_{2}}$ is weak, it is possible to increase $v_{2}$. However, if this confidence level is high, it is possible to decrease $v_{2}$. In a persuasive dialogue, the idea behind the risk of failure is to promote arguments whose hypotheses have more chance to be accepted. Other 
approaches like those used in fuzzy systems to reason with uncertainty (using for example probabilities) can also be used to evaluate the risk of an argument. The advantage of our approach is that it is easy to implement and it reflects the intuitive idea that adding uncertain hypotheses increases the risk of failure of an argument.

The relevance ordering relation denoted by $\preceq_{r}$ can be defined as follows.

Definition 13 (Relevance Ordering Relation). Let $C_{A g_{1}, A g_{2}}=$ $\left\langle S, s, \mathcal{P}_{A g_{1}, A g_{2}}, K D\right\rangle$ be a conversation context and $(H, h)$ and $\left(H^{\prime}, h^{\prime}\right)$ be two relevant arguments in the context $C_{A g_{1}, A g_{2}} \cdot\left(H^{\prime}, h^{\prime}\right)$ is more relevant than $(H, h)$ denoted by $(H, h) \preceq_{r}\left(H^{\prime}, h^{\prime}\right)$ iff:

- $\operatorname{risk}\left(\left(H^{\prime}, h^{\prime}\right)\right)<\operatorname{risk}\left(\left(H^{\prime}, h^{\prime}\right)\right)$ or

- $\operatorname{risk}\left(\left(H^{\prime}, h^{\prime}\right)\right)=\operatorname{risk}\left(\left(H^{\prime}, h^{\prime}\right)\right)$ and $(H, h) \prec_{f a v}^{\mathcal{P}_{A g_{1}, A g_{2}}}\left(H^{\prime}, h^{\prime}\right)$ or

- $\operatorname{risk}\left(\left(H^{\prime}, h^{\prime}\right)\right)=\operatorname{risk}\left(\left(H^{\prime}, h^{\prime}\right)\right)$ and $(H, h) \preceq_{f a v}^{\mathcal{P}_{A g_{1}, A g_{2}}} \quad\left(H^{\prime}, h^{\prime}\right)$ and $\left(H^{\prime}, h^{\prime}\right) \preceq_{\text {fav }}^{\mathcal{P}_{A g_{1}, A g_{2}}}(H, h)$ and $(H, h) \ll_{\text {pref }}^{A g_{1}}\left(H^{\prime}, h^{\prime}\right)$.

According to this definition, $\left(H^{\prime}, h^{\prime}\right)$ is more relevant than $(H, h)$ if the risk of $(H, h)$ is greater that the risk of $\left(H^{\prime}, h^{\prime}\right)$. If the two arguments have the same risk, the more relevant argument is the more favourable one according to the favourite relation $\prec_{f a v}^{\mathcal{P}_{A g_{1}, A g_{2}}}$. If the two arguments have the same risk and they are equal according to the favourite relation, the more relevant argument is the more preferable one according to the preference relation $\ll_{p r e f}^{A g_{i}}$ where $i \in\{1,2\}$. The two arguments have the same relevance if in addition they are equal according to the preference relation. The ordering relation $\preceq_{r}$ is reflexive, antisymmetric, and transitive. The proof is straightforward from the definition and from the fact that $\ll_{p r e f}^{A g_{i}}$ is an ordering relation (see Definition [10).

Computationally speaking, the arguments selection mechanism is based on: (1) the elimination of irrelevant arguments; (2) the construction of new relevant arguments; (3) the ordering of the relevant arguments using the relevance ordering relation; and (4) the selection of one of the most relevant arguments. This process is executed by each participating agent at each argumentation step at the tactical level. The relevant arguments that are not selected at a step $t_{i}$, are recorded and added to the set of potential arguments $P A$ because they can be used at a subsequent step. The set of potential arguments can be viewed as a stack in which the higher level argument is the most relevant one. A relevant argument constructed at a step $t_{i}$ and used latter at a step $t_{j}$ simulates the backtracking towards a previous node in the argumentation tree and the construction of a new path. The following example illustrates this idea.

\section{Example}

In this example, we present only a part of the argumentation tree, which is sufficient to illustrate the arguments selection mechanism. To simplify the notation, arguments are denoted by $a_{i}$ and $a_{i}^{\prime}(1 \leq i \leq n)$. We assume 
that the conversation subject is $S, A=\left\{A g_{1}, A g_{2}\right\}, K D=\{f, l, q\}$, and $\mathcal{P}_{A g_{2}, A g_{1}}=\{p, d, r\} \cup\{\operatorname{pref}(q, p)\}$ where $f, l, q, p, d$ and $r$ are formulas of the language $L$. The part of the argumentation tree we are interested in starts from a node $n_{i}=\left(A g_{1}, a_{1}\right)$ where $a_{1}=\left(\left\{s, \neg s^{\prime}, s \wedge \neg s^{\prime} \rightarrow u\right\}, u\right)$ and $s, s^{\prime}, u$ are formulas of the language $L$. We also assume that from its knowledge base, agent $A g_{2}$ produces four arguments taking into account the current context $C_{A g_{1}, A g_{2}}=\left\langle S, s, \mathcal{P}_{A g_{1}, A g_{2}}, K D\right\rangle$. These arguments are:

$a_{1}^{\prime}=(\{p, k, p \wedge k \rightarrow \neg s\}, \neg s), a_{2}^{\prime}=(\{q, r, c, q \wedge r \wedge c \rightarrow \neg s\}, \neg s)$, $a_{3}^{\prime}=\left(\left\{\neg d, m, \neg d \wedge m \rightarrow s^{\prime}\right\}, s^{\prime}\right)$, and $a_{4}^{\prime}=\left(\left\{e, c, e \wedge c \rightarrow s^{\prime}\right\}, s^{\prime}\right)$.

Where $p, k, q, r, c, d, m$ and $e$ are formulas of the language $L$. Hence: $P A\left(A g_{2}\right)=$ $\left\{a_{1}^{\prime}, a_{2}^{\prime}, a_{3}^{\prime}, a_{4}^{\prime}\right\}$ ( $P A\left(A g_{2}\right)$ is the set of $A g_{2}$ 's potential arguments).

At this step (step 1), $A g_{2}$ should select the most relevant argument using our relevance ordering relation. In order to do that, $A g_{2}$ should evaluate the risk of failure of these arguments. We assume that $v_{1}=0, v_{2}=0.3, v_{3}=0.5$. Consequently: $\operatorname{risk}\left(a_{1}^{\prime}\right)=0.3+0.5=0.8, \operatorname{risk}\left(a_{2}^{\prime}\right)=0+0.3+0.5=0.8$, $\operatorname{risk}\left(a_{3}^{\prime}\right)=0.7+0.5=1.2, \operatorname{risk}\left(a_{4}^{\prime}\right)=0.5+0.5=1$.

The arguments $a_{1}^{\prime}$ and $a_{2}^{\prime}$ have the same risk of failure. However, because $\operatorname{pref}(q, p) \in \mathcal{P}_{A g_{2}, A g_{1}}$ and according to our evaluation algorithm (algorithm4.2), we obtain: $W_{a_{1}^{\prime} / a_{2}^{\prime}, A g_{1}}^{\mathcal{P}_{A 2}, A}=0$ and $W_{a_{2}^{\prime} / a_{1}^{\prime}}^{\mathcal{P}_{A g_{2}, A g_{1}}}=1$.

Therefore, according to definitions 11 and 13, the four arguments are ordered as follows: $a_{3}^{\prime} \preceq_{r} \quad a_{4}^{\prime} \preceq_{r} \quad a_{1}^{\prime} \preceq_{r} \quad a_{2}^{\prime}$. Consequently, $A g_{2}$ selects $a_{2}^{\prime}$. Then (step 2), $A g_{1}$ should take position on $a_{2}^{\prime}$. For that we assume that $A g_{1}$ has only one argument $a_{2}=(\{f, l, f \wedge l \rightarrow \neg c\}, \neg c)$ attacking $a_{2}^{\prime}$ in the new context $C_{A g_{1}, A g_{2}}=\left\langle S, \neg s, \mathcal{P}_{A g_{1}, A g_{2}}, K D\right\rangle$. Because $f, l \in K D, A g_{2}$ accepts this argument. Thereafter, $\neg c$ is added to $K D$ and according to definition $9, a_{4}^{\prime}$ becomes irrelevant. This argument is removed from the set of $A g_{2}$ 's potential arguments. We then obtain $P A\left(A g_{2}\right)=\left\{a_{1}^{\prime}, a_{3}^{\prime}\right\}$. According to the arguments selection mechanism, $A g_{2}$ selects $a_{1}^{\prime}$ (step 3). Selecting this argument at this step simulates a backtracking towards a lower level node (previous node) in the argumentation tree. This example is illustrated in Fig. 2.

\section{Complexity Analysis}

Having defined an argument selection mechanism, we consider its computational complexity. After briefly recalling some complexity results proved by Parsons and his colleagues [24], which are useful for our framework, we present the complexity results of this mechanism. In addition, We use the polynomial time hierarchy notation as defined in [21]:

$$
\begin{gathered}
\Delta_{0}^{p}=\sum_{0}^{p}=\prod_{0}^{p}=p \\
\text { and } \forall k \geq 0, \Delta_{k+1}^{p}=\mathrm{P}^{\sum_{k}^{p}}, \sum_{k+1}^{p}=\mathrm{NP}^{\sum_{k}^{p}}, \prod_{k+1}^{p}=\mathrm{co}-\sum_{k+1}^{p}
\end{gathered}
$$




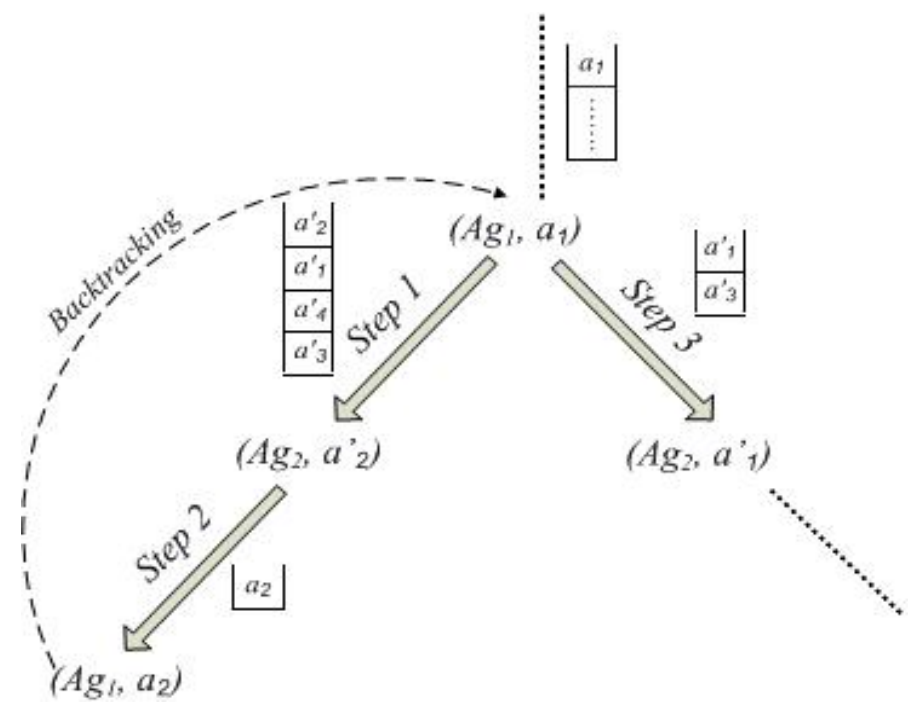

Fig. 2. A part of argumentation tree with the arguments selection mechanism

In particular, $\mathrm{NP}=\sum_{1}^{p}$, co- $\mathrm{NP}=\prod_{1}^{p}, \mathrm{NP}^{\mathrm{NP}}=\sum_{2}^{p}$, co- $\mathrm{NP}^{\mathrm{NP}}=\prod_{2}^{p}$, and $\Delta_{2}^{p}=\mathrm{P}^{\mathrm{NP}}$. According to the results presented in 24, determining if there is an argument for a conclusion $h$ over a knowledge base $\Sigma$ is $\sum_{2}^{p}$-complete. In addition, determining if a given argument is minimal is $\prod_{2}^{p}$-complete.

To determine the complexity of our argument selection mechanism, we have to determine the complexity of the elimination of irrelevant arguments for a given context and the complexity of the relevance ordering relation. This latter is based on three points: (1) the ordering of relevant arguments using the preference relation; (2) the ordering of relevant arguments using the favorite relation; and (3) the risk of failure of an argument. The computational complexity of our strategic and tactic-based reasoning is as follows:

- Elimination of irrelevant arguments. According to Definition 9 an argument $(H, h)$ is irrelevant in the context $C_{A g_{1}, A g_{2}}=\left\langle S, s, \mathcal{P}_{A g_{1}, A g_{2}}, K D\right\rangle$ iff there is no path between the node $\left(A g_{i},(H, h)\right)(i \in\{1,2\})$ of the argumentation tree $T$ and the root of $T$ or; $\exists x \in K D: H \vdash \neg x$. To determine the complexity of this operation, we need the following lemmas:

Lemma 1. Given two arguments $(H, h)$ and $\left(H^{\prime}, h^{\prime}\right)$, determining if $\left(H^{\prime}, h^{\prime}\right)$ attacks $(H, h)$ is co-NP-complete.

Proof. According to Definition 3, an argument $\left(H^{\prime}, h^{\prime}\right)$ attacks $(H, h)$ iff $H^{\prime} \vdash \neg h$. The problem is consequently to decide if $H^{\prime} \rightarrow \neg h$ is a tautology, which is co-NP-complete.

Lemma 2. Given a conversation context $C_{A g_{1}, A g_{2}}=\left\langle S, s, \mathcal{P}_{A g_{1}, A g_{2}}, K D\right\rangle$, and an argument $\left(H^{\prime}, h^{\prime}\right)$, determining if there is a path between the node 
$\left(A g_{i},\left(H^{\prime}, h^{\prime}\right)\right)(i \in\{1,2\})$ of the argumentation tree $T$ and the root of $T$ is in $P^{\| N P}$ (P with parallel queries to $\left.N P\right)$.

Proof. The argumentation tree is built while the conversation proceeds. The root is the first argument supporting the conversation subject $S$. According to Definition 7, to be added in the tree, each new argument should attacks an existing one. Consequently, determining if there is a path between the node $\left(A g_{i},\left(H^{\prime}, h^{\prime}\right)\right)(i \in\{1,2\})$ of the argumentation tree $T$ and the root of $T$ becomes a problem of deciding if there is an argument in the tree attacked by the new argument $\left(H^{\prime}, h^{\prime}\right)$. To solve this problem, we use the following algorithm:

For each argument $(H, h)$ (in the tree) proposed by the interlocutor, decide whether $\left(H^{\prime}, h^{\prime}\right)$ attacks $(H, h)$ or not.

Because the size of the argumentation tree in terms of the number of nodes is polynomially bounded, and because all these verifications can be done in parallel, by Lemma 1, the complexity of this algorithm is in $\mathrm{P}^{\| \mathrm{NP}}$.

Lemma 3. Given a conversation context $C_{A g_{1}, A g_{2}}=\left\langle S, s, \mathcal{P}_{A g_{1}, A g_{2}}, K D\right\rangle$, an argument $(H, h)$, and a formula $x$, determining if $x \in K D \wedge H \vdash \neg x$ is co-NP-complete.

Proof. Because the size of $K D$ is polynomially bounded, deciding if $x \in K D$ is in $\mathrm{P}$. Since $H \vdash \neg x$ is co-NP-complete, the co-NP-completeness of the problem follows.

Theorem 1. Given a conversation context $C_{A g_{1}, A g_{2}}=$ $\left\langle S, s, \mathcal{P}_{A g_{1}, A g_{2}}, K D\right\rangle$, determining whether an argument is irrelevant for $C_{A g_{1}, A g_{2}}$ is in $P^{\| N P}$.

Proof. By Definition 9, to prove that an argument is irrelevant, we have to prove two parts. By Lemma 2, the first part (the existence of the path) is in $\mathrm{P}^{\| \mathrm{NP}}$. It remains to show that the second part is in $\mathrm{P}^{\| \mathrm{NP}}$. For that, we use the following algorithm: For each formula $x \in K D$ check if $H \vdash \neg x$ until all formulas are checked or one formula satisfying $H \vdash \neg x$ is found. Because these verifications can be done in parallel, By Lemma 3, this algorithm is in $\mathrm{P}^{\| \mathrm{NP}}$.

- Ordering of relevant arguments using the preference relation

For the preference relation we suppose that the knowledge base of the agent is stratified. By Definition 10, an argument $\left(H^{\prime}, h^{\prime}\right)$ is more preferable than an $\operatorname{argument}(H, h)$ iff level $\left(H^{\prime}\right) \leq \operatorname{level}(H)$. Therefore, this problem can be solved in a polynomial time.

- Ordering of relevant arguments using the favorite relation

By Definition 11, an argument $\left(H^{\prime}, h^{\prime}\right)$ is more favourable than an argument $(H, h)$ if the weight of $\left(H^{\prime}, h^{\prime}\right)$ is greater than that of $(H, h)$. The evaluation 
of the weight of an argument $(H, h)$ compared to an argument $\left(H^{\prime}, h^{\prime}\right)$ is in $O\left(|H| \times|H|^{\prime}\right)$. Therefore, the complexity of deciding if an argument is favorite than an other one is polynomial.

\section{- Risk of failure of an argument}

By Definition 12, the complexity of determining the risk of an argument is polynomial.

Finally, we can conclude that the complexity of the strategic and tactic reasoning in the worse case is in $\Delta_{2}^{p}$. Consequently, this mechanism is not an additional source of complexity when reasoning with arguments which is in $\sum_{2}^{p}$.

\section{Related Work and Conclusion}

Recently, some interesting proposals have addressed the strategic reasoning of argumentative agents. In [27, Rahwan et al. propose a set of factors that may influence the strategy design. These factors are considered in our framework as operational constraints and criterions. In [2], Amgoud and Maudet define the strategy as a function allowing agents to select a communicative act from the permitted acts. This definition does not take into account the underlying factors and the operational selection mechanism. The more complete framework in the literature addressing tactic and strategic issues of agent communication was developed by Kakas et al. [15]. The authors propose an argumentation-based framework encompassing private tactics of the individual agents and strategies that reflect different classes of agent attitudes. This framework uses sceptical and credulous forms of argumentative reasoning. Private strategies specify the dialogue moves an agent is willing to utter, according to its own objectives and other personal characteristics. Unlike our proposal, this work does not specify the relation between strategy and tactic. In addition, strategies and tactics are mainly represented using a preference policy on the dialogue moves. However, our strategy and tactic theory is based on the goals and sub-goals agents want to achieve. The context notion we use in our framework that reflects the conversational goal and the different agents' beliefs is different from the one used by the authors, which is generally defined on the basis of some priority rules.

The different proposals that have considered the strategic level, have neglected the important relation between strategy and tactics. The contribution of this paper is the proposition of an approach allowing agents to combine strategic and tactic reasoning in order to be more efficient in their communications. The link between strategic and tactic levels enables agents to have global and local visions of the dialogue. In addition, our tactic theory provides a strong mechanism to select the most appropriate argument depending on the strategy adopted by the agent. The mechanism uses our relevance principle that takes into account the conversation context. This selection mechanism is implemented in the case of persuasion dialogues using logical programming and an agent-oriented platform (Jack Intelligent Agents). In addition, an important advantage of our approach is the fact that it allows backtracking. 
The approach presented in this paper is general and can be implemented for other dialogue types. As future work, we plan to define in a systematic way the relevance ordering for each dialogue type. In addition, we intend to enhance protocols based on dialogue games with our strategic and tactic approach. This will allows us to develop more flexible and efficient argument-based agent conversations. We also intend to analyze and evaluate the behavior of the proposed heuristics (e.g. the notion of risk of failure). On the other hand, our framework is operational in its design. Thus, if it is different from the one developed by Sadri et al. [30, which is more declarative. Considering the declarative meaning and investigating the formal properties of our argumentation setting is another key issue for future work.

Acknowledgements. This work is partially supported by the Natural Science and Engineering Research Council of Canada (NSERC) and by the Fonds Québécois de la Recherche sur la Société et la Culture (FQRSC). Also, the second author is supported by the Faculty of Engineering \& Computer Science of Concordia University (Start-Up Grant). We also would like to thank the three anonymous reviewers for their helpful and interesting comments and suggestions that enable us to improve the quality of this paper.

\section{References}

1. Amgoud, L., Maudet, N., Parsons, S.: Modelling dialogues using argumentation. In: Proc. of the 4th Int. Conf. on Multi-Agent Systems, pp. 31-38. IEEE Press, Los Alamitos (2000)

2. Amgoud, L., Maudet, N.: Strategical considerations for argumentative agents (preliminary report). In: Proc. of the 9th Int. Workshop on Non-Monotonic Reasoning, pp. 409-417 (2002)

3. Atkinson, K., Bench-Capon, T., McBurney, P.: A dialogue game protocol for multiagent argument over proposals for action. Journal of AAMAS. Special issue on Argumentation in Multi-Agent Systems 11(2), 153-171 (2005)

4. Bentahar, J.: A pragmatic and semantic unified framework for agent communication. PhD Thesis, Laval University, Canada (May 2005)

5. Bentahar, J., Moulin, B., Chaib-draa, B.: Commitment and argument network: a new formalism for agent communication. In: Dignum, F.P.M. (ed.) ACL 2003. LNCS (LNAI), vol. 2922, pp. 146-165. Springer, Heidelberg (2004)

6. Bentahar, J., Moulin, B., Meyer, J-J.Ch., Chaib-draa, B.: A logical model for commitment and argument network for agent communication. In: Proc. of the 3rd Int. Joint Conf. on AAMAS, pp. 792-799 (2004)

7. Bentahar, J., Moulin, B., Meyer, J-J.C., Lespérance, Y.: A new logical semantics for agent communication. In: Proc. of the 7th Int. Workshop on Computational Logic in Multi-Agent Systems 2006 (accepted)

8. Besnard, P., Hunter, A.: A logic-based theory of deductive arguments. Artificial Intelligence 128, 203-235 (2001)

9. Chesñevar, C.I., Maguitman, A., Loui, R.: Logical models of argument. ACM Computing Surveys 32, 337-383 (2000)

10. Dignum, V.: A model for organizational interaction: based on agents, founded in logic. PhD Thesis, Utrecht University, The Netherlands (2004) 
11. Dung, P.M., Kowalski, R.A., Toni, F.: Dialectic proof procedures for assumptionbased, admissible argumentation. Artificial Intelligence 170(2), 114-159 (2006)

12. Flieger, J.C.: Relevance and minimality in systems of defeasible argumentation. Internal Report. Imperial College of Science, Technology and Medecin (2002)

13. Goldratt, E.: Theory of Constraints. North River Press (1999)

14. Kakas, A., Moraitis, P.: Argumentation based decision making for autonomous agents. In: Proc. of the 2nd Int. Joint Conf. on AAMAS, pp. 883-890 (2003)

15. Kakas, A., Maudet, N., Moraitis, P.: Layered strategies and protocols for argumentation-based agent interaction. In: Rahwan, I., Moraïtis, P., Reed, C. (eds.) ArgMAS 2004. LNCS (LNAI), vol. 3366, pp. 64-77. Springer, Heidelberg (2005)

16. Maudet, N., Chaib-draa, B.: Commitment-based and dialogue-game based protocols: new trends in agent communication languages. The Knowledge Engineering Review 17(2), 157-179 (2002)

17. Moulin, B., Irandoust, I., Bélanger, M., Desbordes, G.: Explanation and argumentation capabilities: towards the creation of more persuasive agents. Artificial Intelligence Revue 17(3), 169-222 (2002)

18. McBurney, P., Parsons, S., Wooldridge, M.: Desiderata for agent argumentation protocols. In: Proc. of the 1st Int. Joint Conf. on AAMAS, pp. 402-409 (2002)

19. McBurney, P., van Eijk, R.M., Parsons, S., Amgoud, L.: A dialogue game protocol for agent purchase negotiations. Journal of AAMAS 7(3), 235-273 (2003)

20. Moore, D.: Dialogue game theory for intelligent tutoring systems. PhD Thesis, Leeds Mitropolitan University, England (1993)

21. Papadimitriou, C.H.: Computational Complexity. Addison-Wesley, Reading (1994)

22. Parsons, S., Wooldridge, M., Amgoud, L.: On the outcomes of formal inter-agent dialogues. In: Proc. of the 2nd Int. Joint Conf. on AAMAS, pp. 616-623 (2003)

23. Parsons, S., McBurney, P., Wooldridge, M.: Some preliminary steps towards a meta-theory for formal inter-agent dialogues. In: Rahwan, I., Moraïtis, P., Reed, C. (eds.) ArgMAS 2004. LNCS (LNAI), vol. 3366, pp. 1-18. Springer, Heidelberg (2005)

24. Parsons, S., Wooldridge, M., Amgoud, L.: Properties and Complexity of Some Formal Inter-agent Dialogues. J. Log. Comput. 13(3), 347-376 (2003)

25. Prakken, H., Vreeswijk, G.: Logics for defeasible argumentation, 2nd edn. Handbook of Philosophical Logic (2000)

26. Rahwan, I., Ramchurn, S.D., Jennings, N.R., McBurney, P., Parsons, S., Sonenberg, L.: Argumentation-based negotiation. The Knowledge Engineering Review 18(4), 343-375 (2003)

27. Rahwan, I., McBurney, P., Sonenberg, L.: Towards a theory of negotiation strategy (a preliminary report). In: Proc. of the Workshop on Game Theoretic and Decision Theoretic Agents (2003)

28. Reed, C., Walton, D.: Towards a Formal and Implemented Model of argumentation schemes in Agent Communication. In: Rahwan, I., Moraïtis, P., Reed, C. (eds.) ArgMAS 2004. LNCS (LNAI), vol. 3366, pp. 19-30. Springer, Heidelberg (2005)

29. Rovatsos, M., Rahwan, I., Fischer, F., Weiss, G.: Adaptive strategies for practical argument-based negotiation. In: Proc. of the 2nd Int. Workshopon on Argumentation in Multi-Agent Systems (2005)

30. Sadri, F., Toni, F., Torroni, P.: Dialogues for negotiation: agent varieties and dialogue sequences. In: Meyer, J.-J.C., Tambe, M. (eds.) ATAL 2001. LNCS (LNAI), vol. 2333, pp. 405-421. Springer, Heidelberg (2002)

31. Searle, J.R.: Speech acts: an essay in the philosophy of languages. Cambridge University Press, England (1969) 
32. Singh, M.P.: A social semantics for agent communication languages. In: Dignum, F.P.M., Greaves, M. (eds.) Issues in Agent Communication. LNCS(LNAI), vol. 1916, pp. 31-45. Springer, Heidelberg (2000)

33. van Dijk, T.A., Kintsch, W.: Strategies of Discourse Comprehension. Academic Press, New York (1983)

34. van Rooy, R.: Relevance of communicative acts. In: Proc. of TARK VIII, pp. 83-96 (2001) 